\title{
Characterization and optimization of Seals-Off for Very Low Pressure Sensors (VLPS) Fabricated by CMOS MEMS Process
}

\author{
Muhamad Ramdzan Buyong ${ }^{1, a}$, Norazreen Abd Aziz ${ }^{1, b}$ Burhanuddin Yeop \\ Majlis ${ }^{1, c}$ \\ ${ }^{123}$ Institute of Microengineering and Nanoelectronics (IMEN) \\ Universiti Kebangsaan Malaysia \\ 43600 Bangi, Selangor, Malaysia \\ amuhdramdzan@yahoo.com, bazreen@vlsi.eng.ukm.my, 'burhan@vlsi.eng.ukm.my
}

\begin{abstract}
Keywords: Bulk micromachining, surface micromachining, CMOS MEMS compatible, CMOS MEMS product.
\end{abstract}

\begin{abstract}
In the world of MEMS processing today, fabrications of membrane are performed using bulk micromachining (BMM). However these techniques not easiest to integrate with CMOS standard process due to not compatible of the processing flow. An attractive alternative deployment of surface micromachining (SMM). There is a trend to use surface micromachining to their advantage of simplicity in design and fabrication process compatibility. This paper presents process development of thin layer membrane for very low capacitive pressure sensor application. The structure of the membrane consists of parallel plate which both top and bottom electrodes were fixed at both sides. Utilizing CMOS MEMS process compatible fabrication of the thin layer membrane involved in three stages; i) hole opening etch, ii) sacrificial intermediate oxide release etch and iii) closing of etch holes. Therefore seals-off process characterization and optimization experiment are presented in this paper, will spur advancement in the development of a CMOS MEMS product for very low capacitive pressure sensor.
\end{abstract}

\section{Introduction}

This work presents an invention of CMOS-based MEMS very low pressure sensor for measuring intraocular pressure inside the eye. Measurement of the cornea and lens interface pressure emphasizes a number of important applications. Abnormal pressure may indicate instability in intraocular pressure. The need to measure the intraocular pressure is for screening the risk of glaucoma that causes damage to the optic nerve which can gradually leading to vision loss. As the current Tonometer in the market exhibit many limitations, a new sensors design based on the more promising CMOS-based MEMS sensor technology was therefore explored. A very low pressure sensor has been proposed to be used in the eye for glaucoma treatment in the course of pressure ranging from $10 \mathrm{mmHg}$ to $75 \mathrm{mmHg}$ [1]. Measurements of the intraocular pressure are usually formed as single measurement at a certain time of the day. In some cases glaucoma patients are hospitalized to measure intraocular pressure more often. However, the resulting intraocular pressure profile consists of 24 to 48 data points a day and is obtained under artificial condition of hospitalization.

Appropriate to miniaturization and integration the beneficial of user such as patient's mobility for continuance of daily activities and continuous measurement for data analysis. Utilizing CMOS compatible process the advantages of thin layer membrane through this technique are monolithic integration, the capability to use standard process steps of integrated circuit fabrication. Thus allocate to integration of read output or input circuit, signal conditioning electronics and wireless communication system. The principal goal of the project was to develop thin layer membrane for very low pressure sensor application. Specific goals to used fabrication technology of CMOS compatible material and were processed in the same as tool as CMOS lot facility $[2,3,4]$. 


\section{Conceptual Design}

The schematic of cross-sectional view of a very low capacitive pressure sensor is shown in Figure 1. The structure of the pressure sensor consists of parallel plate which both top and bottom electrodes were fixed at both sides. The top electrode acts as a thin layer membrane and fixed with the bottom electrode which has dielectric material is absolute air gap, interconnected with contact and metal pad. The working principle of this sensor is based on the capacitances properties that exist between two conductive electrode surfaces. Changes in the distance between the electrode surfaces change the capacitances. This capacitive pressure sensor will be used to indicate changes in position of an air gap due to external force on the thin layer flexible membrane. The geometric dimension of the very low pressure sensor membrane is $100 \mu \mathrm{m}^{2}$ to $1000 \mu \mathrm{m}^{2}$.

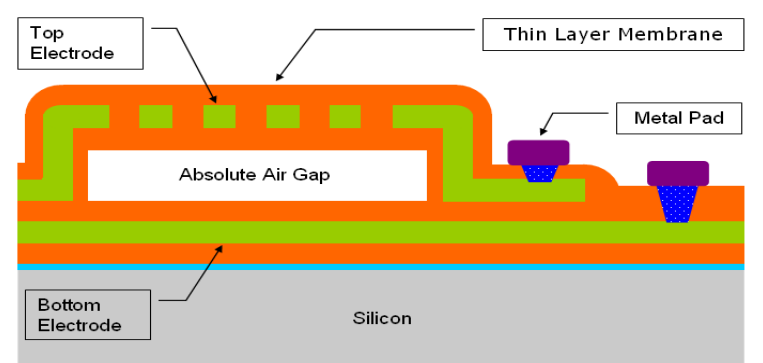

Fig. 1. A cross section schematic membrane of a surface micromachining, very low pressure sensor.

\section{Process challenge}

The challenge in this research is to fabricate the membrane part of the capacitive pressure sensors whereby a sealed cavity (air gap) sandwiched between the two electrodes need to be constructed. The membrane structure is deployed by surface micromachining method using polysilicon as the structural layer together with embedded sacrificial oxide layer which will be removed later. The process development of the thin layer membrane structure was done in three stages:

a. Optimization of anisotropic etching process for opening etch access window.

b. Optimization of isotropic etching process for sacrificial oxide removal.

c. Optimization of nitride deposition for seal-off the opening etches windows.

Fig. 3 Process flow of the thin layer membrane utilizing multi etch access windows (a) access window lithography pattern on polysilicon film (b) dry etching of polysilicon and nitride films for opening of etch windows (c) sacrificial oxide removal by wet etching (d) sealing off etch windows by nitride deposition.
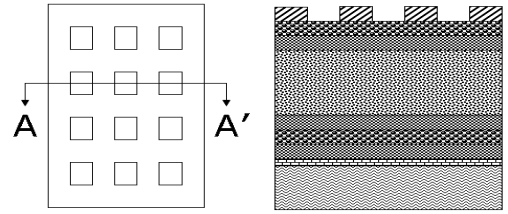

(a)

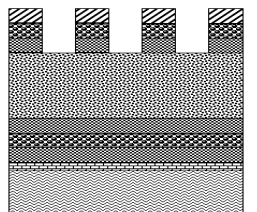

(b)

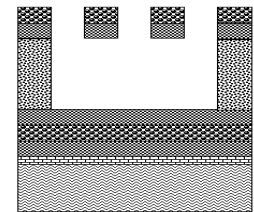

(c)

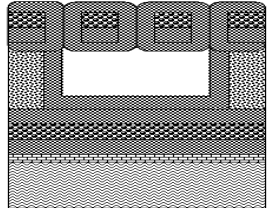

(d)

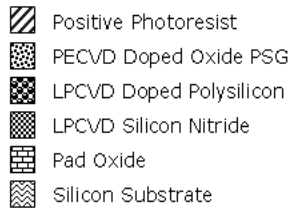

Silicon Substrate

Fig. 2 Proposed process flow of the thin layer membrane 


\section{Fabrication Process}

\section{Characterizations process of seals-off}

The fabricated sample was characterized for the etch holes opening size, nitride deposition method and thickness of the nitride deposition for closing of the etch hole to produce a completely absolute thin layer membrane. Low pressure chemical vapor deposition (LPCVD) deposition mechanism is the most appropriate method to be used for sealed off the etch access windows. This is due to the fact that the LPCVD of low stress silicon nitride has better surface conformality as diffusive process. Furthermore perfect sealed off interior and exterior membrane is achieved for 0.8 $\mu \mathrm{m}$ hole opening etch with $5000 \AA$ of LPCVD low stress silicon nitride of $2.0 \mu \mathrm{m}$ of height thin layer membrane. Fig. 3, Focus ion beam (FIB) images presented below show behavior of LPCVD low stress silicon nitride.

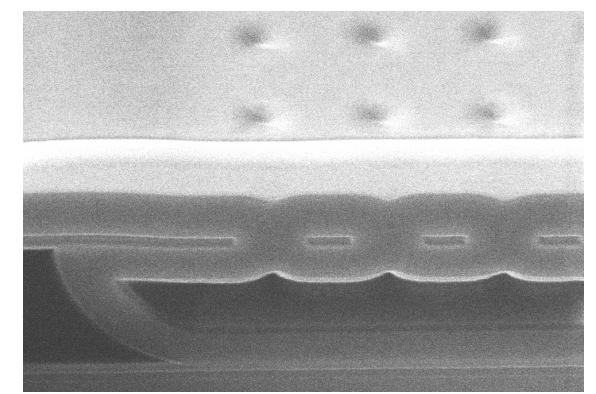

Fig. 3. FIB image of LPCVD sealed, $0.8 \mu \mathrm{m}$ hole opening and sealed with $5000 \AA \AA$ LPCVD

Plasma-enhanced chemical vapor deposition (PECVD) deposition is only works on one part, the top side, which due to the fact that nature deposition mechanism of PECVD is a mass transport limited thus it deposits on top of the structure only. PECVD silicon nitride has the tendency to deposit vertically to the bottom of the membrane through the etching opening access window, thus not capable to seal off the window opening. Effort to reduce the etch holes opening size and density with increase the thickness of the PECVD proved unsuccessful as further stack of silicon nitride permanently reside the membrane and this will adversely affects its performance. Fig. 4, FIB images presented below show behavior of PECVD silicon nitride.

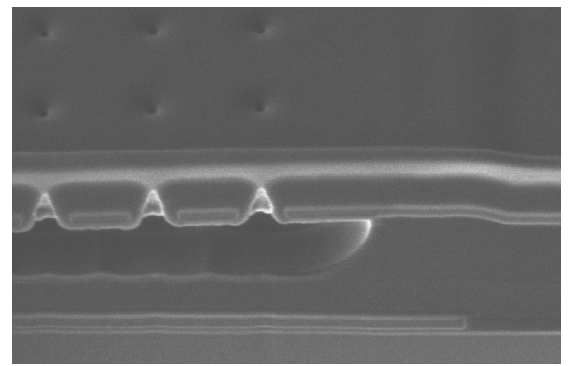

Fig. 4. FIB image of PECVD sealed, $0.8 \mu \mathrm{m}$ hole opening and sealed with $6000 \AA \AA$ PECVD

\section{Optimizations process of seals-off}

In this work, as increased LPCVD Nitride seal off thickness will decrease the etch opening hole. Within the first run optimization study, $0.8 \mu \mathrm{m}$ of etch opening hole is reduced to $0 \mu \mathrm{m}$ as increased LPCVD Nitride from $0 \AA$ to $5000 \AA$. In order to find optimum thickness for sealing process, we visualized the sealing surface area. Since LPCVD silicon nitride based on diffusion process, means that the deposition of surface reaction is identical. We have made assumption that optimum sealed is achieved when the thickness of interior (top, sidewall and bottom inner sealed) and exterior (top membrane sealed) is identical. If the deposition thickness of interior and exterior is less than half 
holes opening sizes we not able to seal the holes opening and if the top membrane exterior thick than interior it show over-sealed. Fig. 5, scanning electron microscope (SEM) images presented below it can be seen that the top, sidewall and bottom inner sealed has comparable thickness. Mean that the maximum sealing thickness is $5000 \AA$ for $0.8 \mu \mathrm{m}$ holes opening. However PECVD silicon nitride has no capability to sealed-off the holes opening as shows at Fig. 6, in fact the actual PECVD sealed thickness is $6000 \AA$.
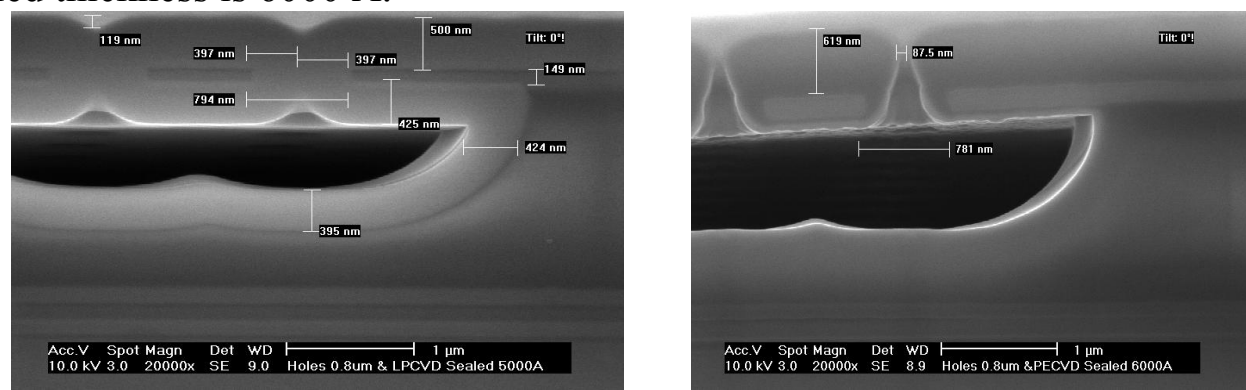

Fig. 5, SEM image of LPCVD sealed with 5000 Å and Fig. 6, PECVD sealed with $6000 \AA$.

Further experiments have been performed to investigate the seals off process and to verify the process repeatability and reliability through opening holes of 0.8 and $1.0 \mu \mathrm{m}$. The sealed thickness of LPCVD low stress silicon nitride is ascending from $0 \AA$ to $6000 \AA$. By referred FESEM images measurement by "movie check", we plot the graph for holes opening and sealed thickness to understand details sealing behavior. For opening holes of 0.8 and $1.0 \mu \mathrm{m}$ the optimum sealed is accomplish when the sealed thickness is $4000 \AA$ and $5000 \AA$. Based on plotted data we concluded the ratio of holes opening to sealed thickness is $1: 0.5$.
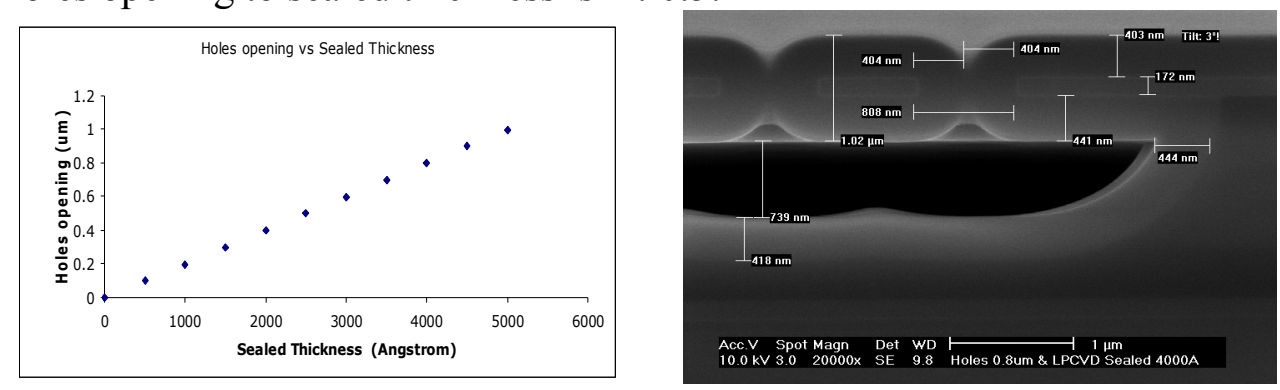

Fig. 7, Shows the co-relation graph between holes opening with sealed thickness. Fig. 8, SEM image of LPCVD sealed, $0.8 \mu \mathrm{m}$ hole opening and sealed with $4000 \AA$ LPCVD silicon nitride.

\section{Summary}

Overall fabrication process step necessity well-matched with CMOS baseline manufacturing processes so that they not contaminate those processes. This integration promises to improve the performance of micromechanical devices as well cost manufacturing, packaging and instrumenting these devices by combining the micromechanical devices with microelectronic subsystem in the same manufacturing and packaging process.

\section{References}

[1] Lawrence J. Schwartz, MD “Glaucoma” http://www.medicinenet.com/

[2]Gunther Zimmer United States Patent 5,431,057 (1995) “Integratable Capacitive Pressure Sensor and Process for its Manufacture"

[3]Henry Guckel United States Patent 4,744,863 (1988) “Sealed Cavity Semiconductor Pressure Transducers and Method of Producing the same".

[4]H. Berney "Critical Processing Issues for Micromachined Sacrificial Layer Etching and Sealing”, Sensor and Actuators 76 (1999) 356-364 